\title{
Effect of band filling on symmetric and asymmetric one-dimensional periodic Anderson model
}

\author{
Yan Luo and Nicholas Kioussis \\ Department of Physics, California State University Northridge, Northridge, California 91330-8268
}

(Received 29 November 2001; published 29 April 2002)

\begin{abstract}
We have studied the ground-state properties of the symmetric and asymmetric one-dimensional periodic Anderson model as a function of band filling and the model parameters $\left(V, U\right.$, and $\left.E_{f}\right)$ using the local mean-field (LMF) approach. We have constructed the phase diagram for both cases and have calculated the energy, local moment, and occupation number of the $f$ level. The LMF approach can reveal the basic properties of the system throughout the entire parameter space. For the symmetric case $\left(E_{f}=-U / 2\right)$, close to quarter and half filling the antiferromagnetic phase is the ground state. For intermediate band-filling there is a second-order phase transition to a ferromagnetic state. At quarter filling there is a transition from a metallic paramagnetic state to an insulating antiferromagnetic state as the on-site Coulomb interaction increases. The asymmetric case is treated in the mixed-valence regime $\left(E_{f}=0\right)$. At half filling the ground state is antiferromagnetic while at or close to quarter filling it is paramagnetic. Below half filling the ground state becomes ferromagnetic, suggesting that doping destroys rapidly the antiferromagnetic $f-f$ spin correlations. For small values of $U$ we find a paramagnetic state at all band fillings. Results for the average occupation number of the $f$ level compare well with density matrix renormalization group calculations.
\end{abstract}

DOI: 10.1103/PhysRevB.65.195115 PACS number(s): 71.27.+a, 71.28. + d, 75.20.Hr, 71.10.Hf

\section{INTRODUCTION}

The lanthanides and actinides, and their compounds, exhibit a great variety of unusual and interesting properties. ${ }^{1}$ Some of the most diverse physical characteristics are found in the heavy-fermion (HF) and intermediate valence (IV) systems. ${ }^{2}$ The magnetic phase diagrams of these materials are quite extraordinary in their large variety of different phases, ranging from simple paramagnetic or Kondo states to various kinds of magnetic ordering. ${ }^{1,2}$ The wide variety of ground states is associated with the partial delocalization of the $4 f$ or $5 f$ states and the hybridization of the $f$-electron ions with the non- $f$-band electrons.

The periodic Anderson model (PAM) is believed to contain the essential physics of this class of strongly correlated electron systems. ${ }^{1}$ The PAM describes a localized $f$ band of strongly correlated electrons hybridized with a conduction (c) band of uncorrelated conduction electrons. As the temperature, band filling, and Hamiltonian parameters are varied, there is an interplay between the Ruderman-KittelKasuya-Yosida (RKKY) interactions ${ }^{3}$ and the Kondo effect. ${ }^{4}$ The RKKY interaction favors ordering the localized $f$ magnetic moments, while the Kondo effect screens the localized magnetic moments and quenches the magnetic interaction through the formation of singlets between the two bands. Therefore, it is important to understand the electronic and magnetic properties of the ground state of the simplest onedimensional Anderson lattice model as a function of the model parameters, namely, the on-site Coulomb interaction $U$, the hybridization $V$, the position of the localized $f$ level $E_{f}$, and the band filling, $n$.

In the Anderson model, there are two distinct regimes. In the Kondo regime, $V \ll U$ and the energy of the localized band $E_{f}$ lies well below the Fermi energy, the occupation of the localized level is close to one $\left(n_{f} \rightarrow 1\right)$, and hence charge fluctuations are negligible. On the other hand, in the mixedvalence regime, the energy of the localized band lies very close to the Fermi level, charge fluctuations are important and hence the average occupancy of the localized orbital is less than 1. In fact, real materials are more likely to be in the mixed-valence regime.

In the past, most studies of the one-dimensional PAM have treated the symmetric case $\left(E_{f}=-U / 2\right)$ at half filling, $n=2$ (when the number of electrons is twice the number of sites). Monte Carlo (MC) simulations ${ }^{5}$ have shown that the local $f$-electron spins are compensated with other $f$ electrons as well as band electrons leading to a nonmagnetic ground state. However, the MC calculations suffer from the sign problem away from half filling. Steiner et al. ${ }^{6}$ have applied the second-order perturbation theory in the fluctuations around the mean-field result and found good agreement with the Monte Carlo results. Ueda et al. ${ }^{7}$ have shown that the ground state of the symmetric PAM is a singlet at half filling. Using the Gutzwiller approach, Rice and $\mathrm{Ueda}^{8}$ studied the $U=\infty$ case in which doubly occupied states of the localized orbital are forbidden. They found that there is always a ferromagnetic instability (assuming no orbital degeneracy) when the energy of the localized orbital is well below the Fermi surface. In contrast, the standard mean-field slaveboson treatment of the PAM (Ref. 9) gives a paramagnetic solution for any filling in the $U=\infty$ case. Guerrero and $\mathrm{Yu}^{10}$ have used the density-matrix-renormalization-group (DMRG) method ${ }^{11}$ to calculate the charge gap, the spin gap, and the quasiparticle gap for the symmetric and asymmetric PAM at half filling.

Away from half filling, results are more limited. Using the DMRG approach, Guerrero and Noack ${ }^{12}$ mapped out the phase diagram of the one-dimensional PAM for the symmetric case and the $U=\infty$ asymmetric case. At quarter filling and in the strong coupling regime they find an $S=0$ ground state with short-range antiferromagnetic correlations. Möller and Wölfle ${ }^{13}$ used the Kotliar and Ruckenstein slave-boson treatment ${ }^{14}$ to study the symmetric PAM. They found that in the strong-coupling case there is a very narrow antiferromag- 
netic (AF) region near quarter filling and that there is a smooth crossover from AF to ferromagnetic order as the band filling increases. When the number of electrons is equal to the number of sites plus one (quarter filling with one additional electron), the ground state was shown to be ferromagnetic for sufficiently large $U{ }^{15}$

In this paper we study the ground-state properties of the symmetric and asymmetric one-dimensional PAM as a function of the band filling and the model parameters $(V, U$, and $E_{f}$ ) using the local mean-field (LMF) approach. We have previously employed the LMF approach to study the groundstate properties of the symmetric one-dimensional PAM model at half filling. ${ }^{16}$ The results for the total energy, the $f$-magnetic moment, the effective hybridization, and the $f$ and $c$-projected density of states are in very good agreement with Monte Carlo (Ref. 5) results and results using the second-order perturbation theory in the fluctuations. ${ }^{6}$ The good agreement of our results with MC and DMRG results puts the LMF approach on a firmer basis in two and three dimensions, where only a few results are available and other approaches may be limited (for example, there is a sign problem in the $\mathrm{MC}$ calculations away from half filling). Although fluctuation effects are expected to yield corrections to the mean-field results, we expect that the qualitative nature of the mean-field state to be maintained and that the mean-field treatment can reveal the basic properties of the system throughout the entire parameter space (including band filling and temperature) in contrast to other approaches, such as the Gutzwiller approximation, ${ }^{8}$ the large orbital $1 / N$ expansion, ${ }^{17}$ and various perturbative approaches, each making a different set of approximations, and thereby limiting their applicability to small regions of the parameter space.

The paper is organized as follows. In Sec. II we briefly describe the one-dimensional PAM and discuss the main features of the local mean-field (LMF) approach. In Secs. III A and III B we present numerical results and the phase diagram for the symmetric and asymmetric cases, respectively. Our conclusions are given in Sec. IV.

\section{MODEL AND METHOD}

The Hamiltonian for the one-dimensional periodic Anderson model is

$$
\begin{aligned}
H= & -t \sum_{i \sigma}\left(c_{i \sigma}^{\dagger} c_{i+1 \sigma}+\text { H.c. }\right)+E_{c} \sum_{i \sigma} n_{i \sigma}^{c}+E_{f} \sum_{i \sigma} n_{i \sigma}^{f} \\
& +U \sum_{i} n_{i \uparrow}^{f} n_{i \downarrow}^{f}+V \sum_{i \sigma}\left(f_{i \sigma}^{\dagger} c_{i \sigma}+\text { H.c. }\right) .
\end{aligned}
$$

Here, $t$ is the nearest-neighbor hopping matrix element for the conduction electrons, $c_{i, \sigma}^{+}\left(c_{i, \sigma}\right)$ and $f_{i, \sigma}^{+}\left(f_{i, \sigma}\right)$ create (annihilate) Wannier electrons in $c$ - and $f$-like orbitals on site $i$ with spin $\sigma$, respectively. $E_{f}$ and $E_{c}$ are the energy levels of the bare localized orbital and the center of the conduction band, respectively, $V$ is the on-site hybridization matrix element between the local $f$ orbitals and the conduction band and $U$ is the on-site Coulomb repulsion of the $f$ electrons. In this paper we use a simple nearest-neighbor tight-binding model for the conduction band dispersion, $\epsilon_{k}=-2 t \cos k$ $(t=0.5)$ and choose $E_{c}=0$.

We employ a Hartree-Fock type local mean-field approximation, described in detail in Ref. 16, in which the diagonal matrix elements of the Green's functions are calculated using the Anderson's renormalized perturbation expansion method. ${ }^{18}$ Here, we briefly describe the method. The manybody term in Eq. (1) is approximated as

$$
U n_{i \uparrow}^{f} n_{i \downarrow}^{f} \simeq \epsilon_{i \uparrow}^{f} n_{i \uparrow}^{f}+\epsilon_{i \downarrow}^{f} n_{i \downarrow}^{f}-(1 / U) \epsilon_{i \uparrow}^{f} \epsilon_{i \downarrow}^{f} .
$$

Here,

$$
\epsilon_{i, \uparrow}^{f}=U\left\langle n_{i, \downarrow}^{f}\right\rangle, \quad \epsilon_{i, \downarrow}^{f}=U\left\langle n_{i, \uparrow}^{f}\right\rangle,
$$

are the local effective potentials of the $f$-state energy level to be determined self-consistently,

$$
\left\langle n_{i, \sigma}^{f}\right\rangle=\int^{E_{F}} \rho_{i, \sigma}^{f}(E) d E,
$$

is the average number of $f$ electrons with spin $\sigma$ at site $i$, $\rho_{i, \sigma}^{f}(E)$ is the local $f$ partial density of states, and $E_{F}$ is the Fermi energy.

For each magnetically-ordered state the self-consistency iterative scheme starts with plausible initial values for the local potentials $\epsilon_{\uparrow}^{f}, \epsilon_{\downarrow}^{f}$, and then calculating $E_{F}$ for a given value of the number of electrons per site (band filling) $n$ from

$$
n=\int^{E_{F}} \rho(E) d E,
$$

where the total density of states is

$$
\rho(E)=\sum_{\sigma}\left[\rho_{\sigma}^{f}(E)+\rho_{\sigma}^{c}(E)\right] .
$$

Here, $\rho_{\sigma}^{f}(E)$ and $\rho_{\sigma}^{c}(E)$ are the $f$ - and $c$-partial density of states calculated from the diagonal matrix elements of the $f$ and $c$ Green's functions, respectively. The average number of $f$ electrons with $\sigma=\uparrow$ or $\downarrow$ is determined from Eq. (5), and new values for the $\epsilon_{\uparrow}^{f}, \epsilon_{\downarrow}^{f}$ are obtained from Eq. (3). This procedure is repeated until it converges to the self-consistent values of $\left\{\epsilon_{\uparrow}^{f}, \epsilon_{\downarrow}^{f}\right\}$. We have considered the cases of ferromagnetic state $\mu_{i}^{f}=\mu_{i+1}^{f}=\mu_{0}\left(\left\langle n_{i, \uparrow}^{f}\right\rangle-\left\langle n_{i, \downarrow}^{f}\right\rangle\right)$, antiferromagnetic state $\mu_{i}^{f}=-\mu_{i+1}^{f}$ and paramagnetic state $\mu_{i}^{f}=\mu_{i}^{c}=0$.

\section{RESULTS AND DISCUSSION}

\section{A. Symmetric case}

In Figs. 1(a) and 1(b) we show the $U-V$ phase diagram for the half-filled case and the quarter-filled case. For both cases the phase diagram consists of the antiferromagnetic and the paramagnetic phase. As expected with any mean-field treatment, we find a critical value of $U, U_{c}$, below which the state is paramagnetic. In the strong coupling regime ( $U$ $\gg V$ ) the phase is antiferrromagnetic. For the case of the $f$ level well below the conduction band and the Coulomb interaction $U$ large and in the absence of hybridization ( $V$ $=0)$, the ground state at quarter filling has one electron at 


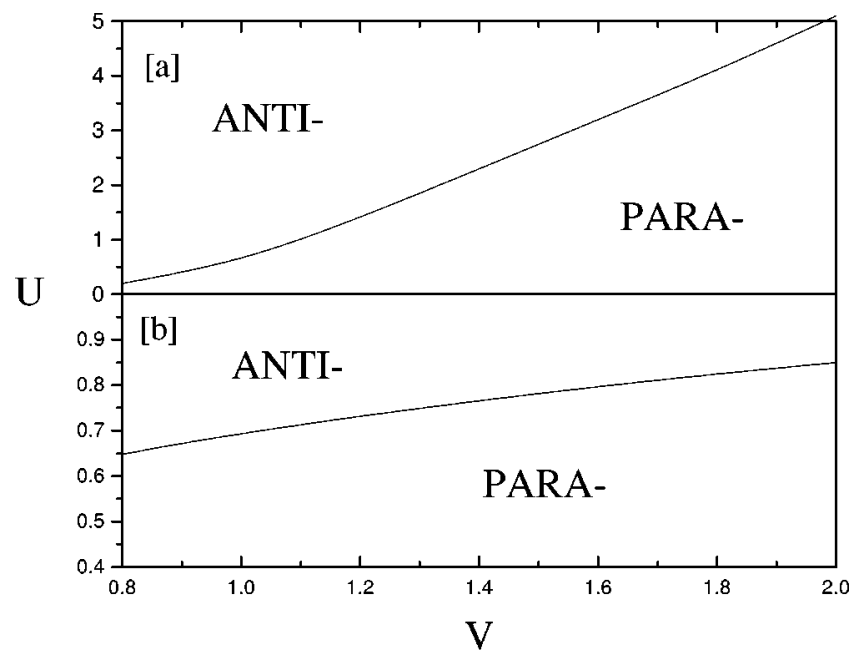

FIG. 1. The $U-V$ phase diagram for the symmetric periodic Anderson model (a) at half filling $(n=2)$ and (b) at quarter filling $(n=1)$.

each $f$ site and there is a degeneracy in the spin configurations. It can be shown using perturbation theory that when $V>0$, exchange interactions remove the degeneracy, and the effective nearest-neighbor interaction favors antiferromagnetic alignment. ${ }^{13}$ The induced conduction-electron local moment $\mu_{c}$ is small $(\sim 0.1)$ and is aligned antiferromagnetically with the local $f$ moment. For a given value of $U$, the $f$ and $c$-local moments decrease with increasing hybridization, until they vanish in the paramagnetic phase. Note, that for $V>1$, the critical value of $U$ is smaller for the quarter-filled case, indicating larger AF correlations than those in the halffilled case. Our results for both the half-filled and quarterfilled cases are in agreement with those of DMRG calculations which show a zero total spin $S$ with short-range antiferromagnetic correlations that increase with increasing $U$. Thus, the DMRG results are in agreement with the LMF results in Fig. 1(b), which show an AF phase ground state for a wider range of hybridization provided that $U$ is large enough for local moment formation.

In Fig. 2 we present the $f$ - and the $c$-electron occupation number as a function of the on-site Coulomb interaction $U$ at quarter-filling for two values hybridization $V=0.375$ (solid curve) and 1.0 (dotted curve). For large $U$, the $n_{f} \rightarrow 1$ and $n_{c} \rightarrow 0$. In view of the transition from the paramagnetic to the antiferromagnetic phase with increasing $U$ in Fig. 1(b), the results in Fig. 2 indicate a transition from a metallic paramagnetic ground state to an insulating antiferromagnetic ordered ground state as $U$ increases.

In Fig. 3, we show the $U$ versus band filling phase diagram of one-dimensional symmetric PAM for hybridization $V$ values of 0.375 (solid curve) and 1.0 (dotted curve), respectively. Close to half filling we find an antiferromagnetic phase for $U>U_{c}$. As the band filling decreases there is a second-order transition from an antiferromagnetic to a ferromagnetic phase (the $f$ moment changes continuously). Closely above quarter filling we find a narrow antiferromagnetic region, in agreement with the results of DMRG calculations. ${ }^{12}$ When the filling is increased from quarter fill-

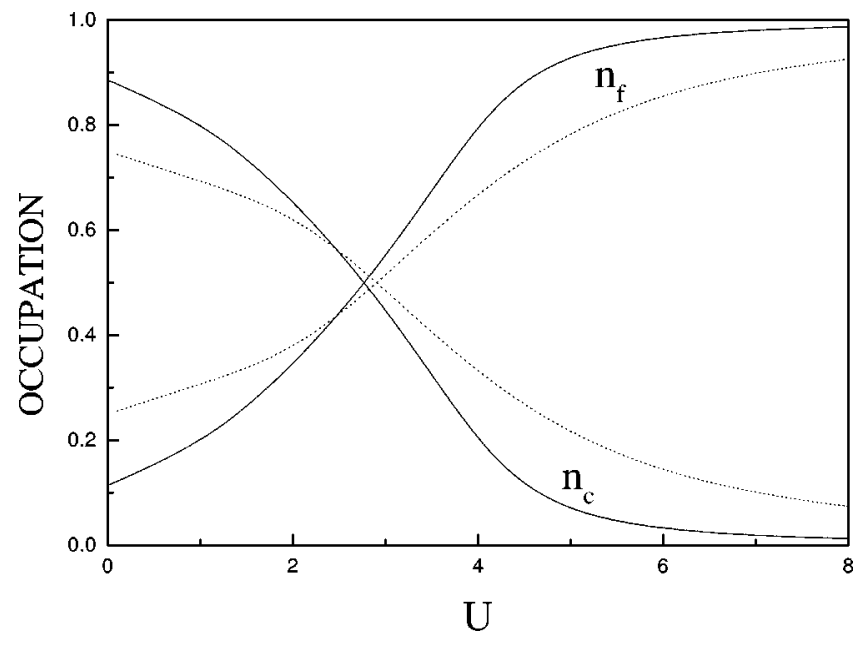

FIG. 2. The $f$-electron and $c$-electron occupation number at quarter filling as a function of $U$ for $V=0.375$ (solid curve) and 1.0 (dotted curve).

ing, the additional electrons go into the conduction orbitals because of the strong Coulomb repulsion $U$ in the $f$ orbitals. In this case, there is an on-site antiferromagnetic correlation between the electron in the conduction orbital and the one in the $f$ orbital, namely, for both the ferromagnetic and antiferromagnetic phases, the local $c$-electron moment is aligned antiferromagnetically with the $f$-electron moment. To optimize the kinetic energy of the conduction electrons, it is favorable for the $f$ electrons to have their spins aligned in the same direction. As expected, for small values of the on-site Coulomb interaction $\mathrm{U}<U_{c}$, the ground state is paramagnetic for all band filling. Increasing the hybridization, stabilizes the ferromagnetic nearest-neighbor $f$ - $f$ correlations over a wider range of $U$ and band filling. The calculated magnetic phase diagram of the symmetric one-dimensional PAM is in good agreement with that calculated by Möller and Wölfle ${ }^{13}$ for the large $U$ case, and that of Guerrero and Noack. $^{12}$

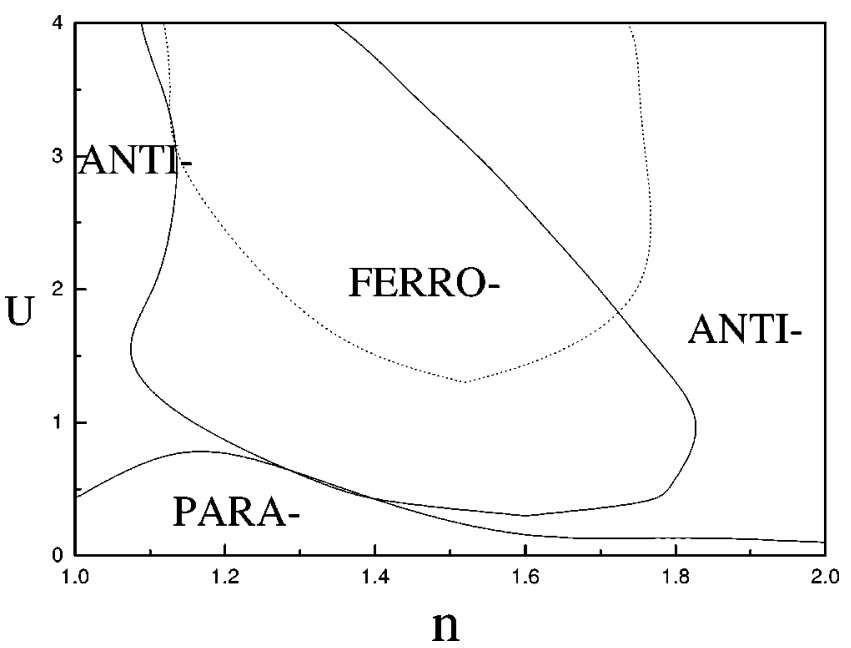

FIG. 3. The $U$ versus band filling phase diagram for the symmetric $\left(E_{f}=-U / 2\right)$ one-dimensional periodic Anderson model for $V=0.375$ (solid curve) and $V=1.0$ (dotted curve). 


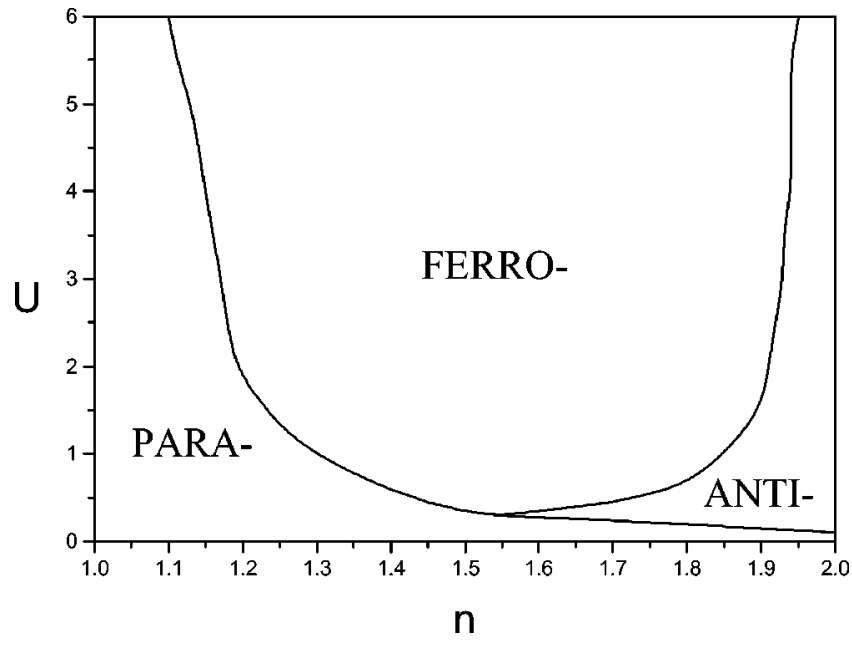

FIG. 4. The $U$ versus band filling phase diagram of the asymmetric periodic Anderson model with $E_{f}=0$ and $V=0.375$.

\section{B. Asymmetric case}

Although the symmetric case is the most heavily studied limit of the Anderson model, most real systems have neither particle-hole symmetry nor $\left\langle n_{f}\right\rangle=1$. For the asymmetric case at half filling, previous results include the DMRG (Ref. 10) and the second-order perturbation theory in the around the mean-field studies, ${ }^{6}$ and away from half filling the DMRG results for the $U=\infty$ case. $^{12}$ In this section we study the behavior of the asymmetric case, i.e., by examining its $U$, $V$, and band filling dependence. We place the $f$ orbital $\left(E_{f}\right.$ $=0)$ at the Fermi energy so that states with no electrons $\left(f^{0}\right)$ and states with one electron $\left(f^{1}\right)$ in the $f$ level are equal in energy, while states with two electrons are suppressed due to the strong Coulomb interaction. In this mixed-valence case, charge fluctuations are allowed and the occupation of the $f$ level $n_{f}<1$.

In Fig. 4 we show the $U$ versus band filling phase diagram for the asymmetric PAM with $E_{f}=0$. At half filling, the ground state is antiferromagnetic for $U>U_{c}$, as in the symmetric case. However, for intermediate band filling and for large $U$ we find that the ground state becomes ferromagnetic, suggesting that doping away from half filling destroys rapidly the antiferromagnetic $f-f$ spin correlations. For small values of $U$ (i.e., smaller than the critical value for the onset of magnetic ordering), the ground state is paramagnetic, as expected. We find a paramagnetic ground state at and slightly above quarter filling $(n=1)$ for all values of $U$. Note, that we find a boundary between the paramagnetic and ferromagnetic ground state for large but finite $U$ and small band filling, in agreement with the slave boson results, ${ }^{9}$ but in contradiction with the DMRG results ${ }^{12}$ which predict that for small values of $E_{f}$ and $U=\infty$ the ground state is paramagnetic at all fillings.

In Fig. 5 we show the average occupation of the $f$ level in the ground state at half filling $(n=2)$ as a function of $U$ for the symmetric (dashed curve) and asymmetric case (solid curve) with $E_{f}=0$. We have set $V=1$ for both cases. The solid circles denote the DMRG results for the asymmetric case for $E_{f}=0$ and $V=1$. In the symmetric case, the occu-

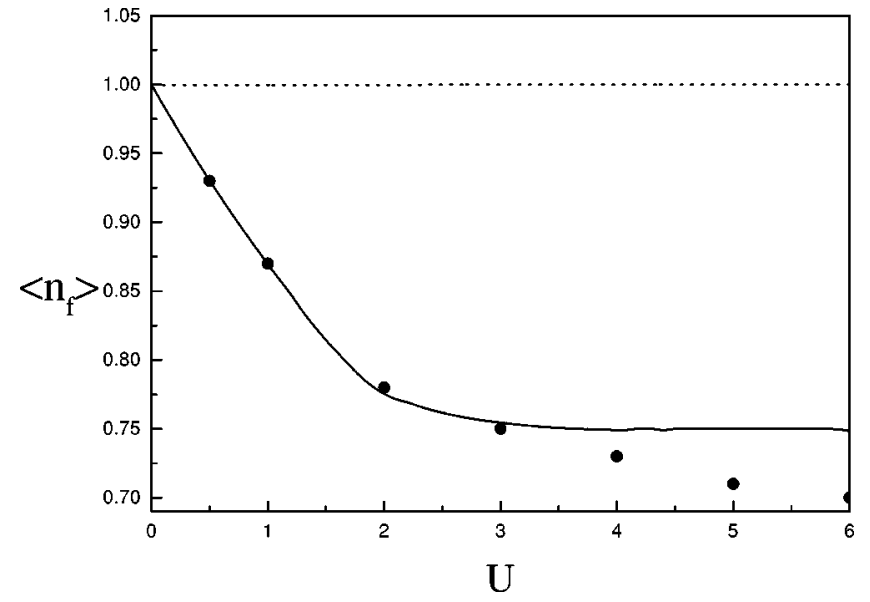

FIG. 5. Occupation $n_{f}$ of the $f$ level versus $U$ at half filling with $V=1$ for the symmetric case $E_{f}=-U / 2$ (dotted curve) and the asymmetric case $E_{f}=0$ (solid curve). The closed circles are the DMRG results for the asymmetric case, with $E_{f}=0$ and $V=1$ (Ref. 10).

pation number of the $f$ level is 1 in the ground state for any Coulomb interaction $U$ due to particle-hole symmetry. However, in the asymmetric case the $f$-level occupation number $n_{f}$ is less that 1 in the mixed-valence regime, due to the charge fluctuations between the $f^{0}$ and $f^{1}$ configurations. We see that $n_{f}$ decrease from 1 towards a value close to 0.75 as $U$ increases. Overall there is a good agreement of the LMF results with those of DMRG, even though the DMRG calculations give $\left\langle n_{f}\right\rangle \rightarrow 0.7$ as $U \rightarrow \infty$. This difference is due to the fact that the LMF approach neglects quantum fluctuations.

The average of the square of the local moment, $\left\langle\mu_{f}^{2}\right\rangle$, at half filling $(n=2)$ is plotted versus $U$ in Fig. 6 for the symmetric (solid curve) and the asymmetric case (dotted case) with $V=0.375$. For the symmetric case $E_{f}=-U / 2$, while $E_{f}=0$ for the asymmetric case. In both cases the ground state is antiferromagnetic. In one dimension there is of course no long-range order. There the LMF description

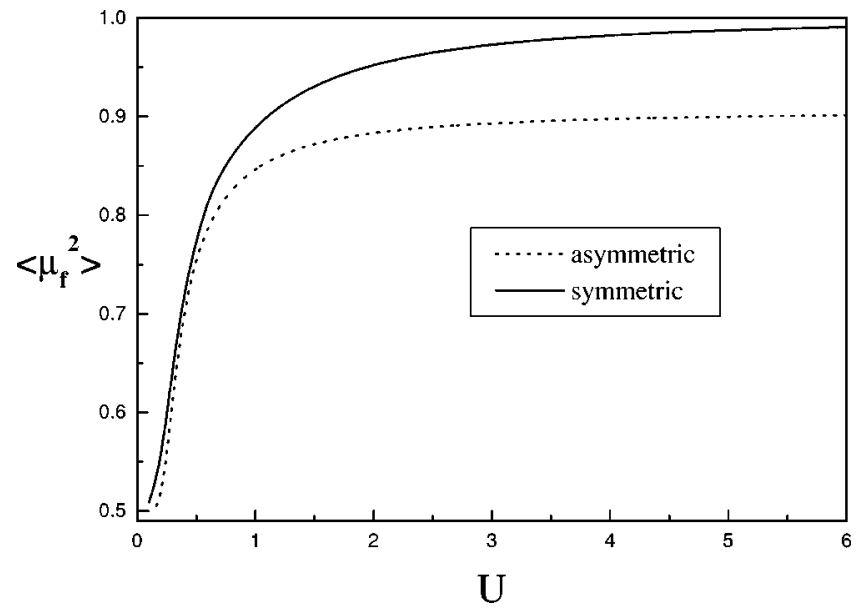

FIG. 6. Square of the local $f$ magnetic moment as a function of $U$ at half filling with $V=0.375$. The solid curve denotes the symmetric case $\left(E_{f}=-U / 2\right)$ and the dotted curve the asymmetric case $\left(E_{f}=0.0\right)$. 


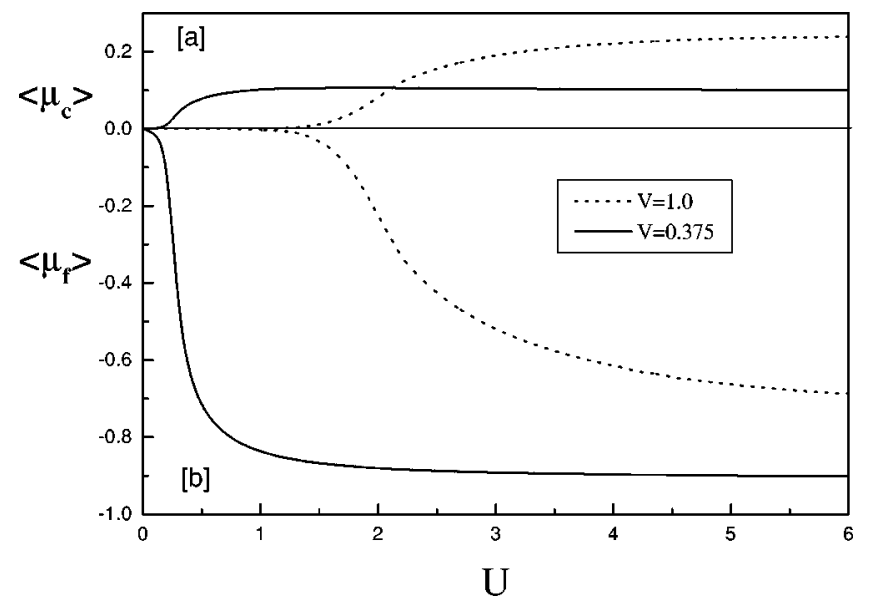

FIG. 7. Local $f$ moment $\mu_{f}$ and conduction-electron moment $\mu_{c}$ versus $U$ at half filling for the asymmetric case $E_{f}=0$, for $V=1.0$ (dotted curve) and $V=0.375$ (solid curve), respectively.

should be taken as simulating short-range magnetic order, which apparently contributes to the ground-state energy in an important way. The square of the local moment in the asymmetric case is obviously suppressed due to the charge fluctuations. The local $f$ moment $\mu_{f}$ and the conduction-electron moment $\mu_{c}$ at half filling are plotted versus $U$ for the asymmetric case $\left(E_{f}=0\right)$ for $V=0.375$ and 1.0 in Figs. 7(a) and 7(b), respectively. For $U<U_{c}$, the local $f$ moment vanishes (paramagnetic state) and $\mu_{f} \rightarrow 1$ for large $U$. Naturally, the value of $U_{c}$ increases as the hybridization increases. The induced conduction-electron moment $\mu_{c}$ is small and is aligned antiferromagnetically with the local $f$ moment. As the hybridization decreases the local $f$ moment is enhanced for a given $U$, due to the enhancement of the RKKY exchange interactions responsible for the antiferromagnetic ordering.

In Fig. 8 we plot the average occupation $\left\langle n_{f}\right\rangle$ of the $f$ level at quarter filling as a function of $U(V=0.375)$ for the symmetric case and for the asymmetric case in the mixed valence regime $\left(E_{f}=0\right)$. At half filling, the LMF ground state is antiferromagnetic for the symmetric case for $U>U_{c}$, where it is paramagnetic for all $U$ values in the asymmetric case. In contrast to the case at half filling (Fig. 5), the $f$-level occupation at quarter filling $(n=1)$ increases in the symmetric case from about 0.1 at $U=0$ approaching the saturation value of 1 for large $U$, while in the asymmetric case the $f$ occupation is small for all values of $U$. Thus, the average $c$ occupation of the conduction orbitals, $\left\langle n_{c}\right\rangle=n-\left\langle n_{f}\right\rangle$, changes, which will in turn change the position of the Fermi energy. The local $f$ moment $m_{f}$ is plotted versus $U$ at quarter filling in Fig. 9 for the symmetric and asymmetric $\left(E_{f}=0\right)$ cases for $V=0.375$. The local $f$ moment vanishes for all values of $U$ for the asymmetric case (paramagnetic state), while for the symmetric case the sublattice magnetization increases with $U$, since the energy of the $f$ level $E_{f}=-U / 2$ shifts well below the Fermi energy and the $\left\langle n_{f}\right\rangle \rightarrow 1$.

In Fig. 10 we show the Fermi energy versus $U$ at quarter filling with $V=0.375$ for the symmetric and asymmetric case $\left(E_{f}=0\right)$. The Fermi energy decreases with increasing $U$ in the symmetric case, because as $U$ increases the energy, $E_{f}$

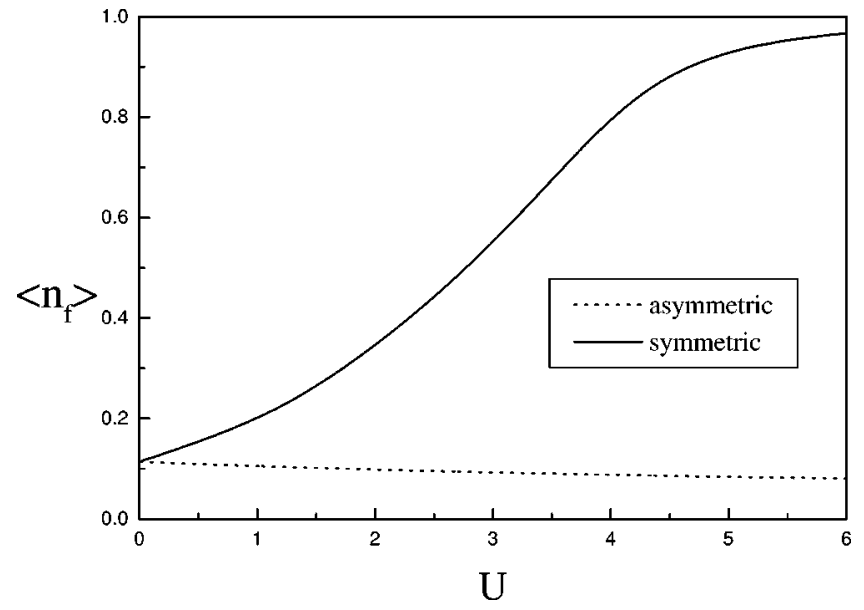

FIG. 8. Occupation $n_{f}$ of the $f$ level versus $U$ at quarter filling and $V=0.375$ for the symmetric $\left(E_{f}=-U / 2\right)$ and the asymmetric case $\left(E_{f}=0\right)$, respectively.

$=-U / 2$, of the localized $f$ state moves away from the Fermi energy, $\left\langle n_{f}\right\rangle \rightarrow 1$, and $\left\langle n_{c}\right\rangle \rightarrow 0$. On the other hand, the Fermi energy slightly increases with $U$ in the asymmetric case. This is due to the fact that $\left\langle n_{f}\right\rangle$ in Fig. 8 decreases slightly with $U$ and hence $\left\langle n_{c}\right\rangle=n-\left\langle n_{f}\right\rangle$ increases slightly with $U$.

\section{CONCLUSION}

We have studied the one-dimensional periodic Anderson model away from half filling using the LMF method. We considered the symmetric Anderson model with $E_{f}=-U / 2$, as well as the asymmetric case in which we set $E_{f}=0$ in order to study the mixed-valence regime. We have constructed the phase diagram for both cases. In the latter case there are more limited results. In contrast to the slave-boson approach applicable only in the limit of large $U$, the LMF approach can reveal the basic properties of the system throughout the entire parameter space.

For the symmetric case, near quarter and half filling we find an antiferromagnetic state in the large $U$ limit. For in-

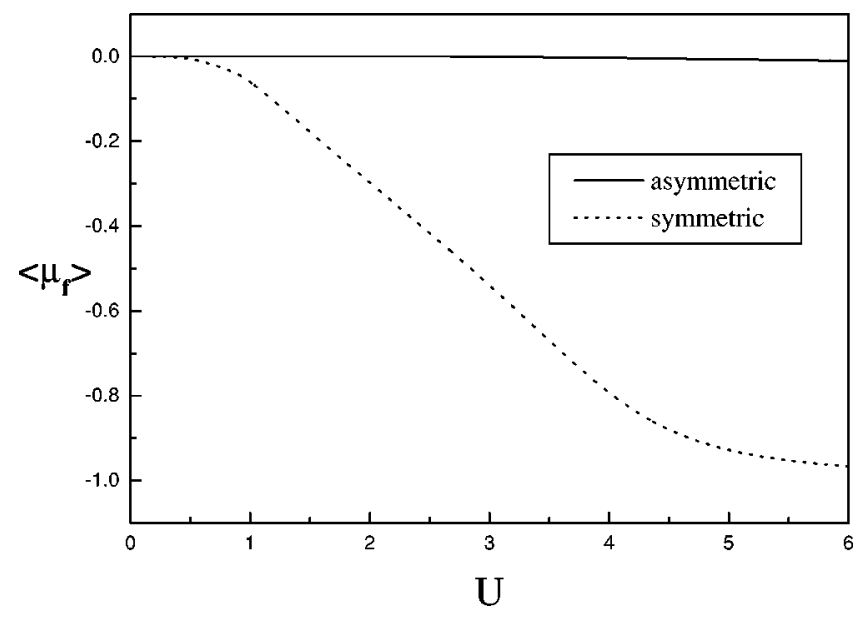

FIG. 9. Local $f$ moment versus $U$ at quarter filling and $V$ $=0.375$, for the symmetric $\left(E_{f}=-U / 2\right)$ and the asymmetric case $\left(E_{f}=0\right)$, respectively. 


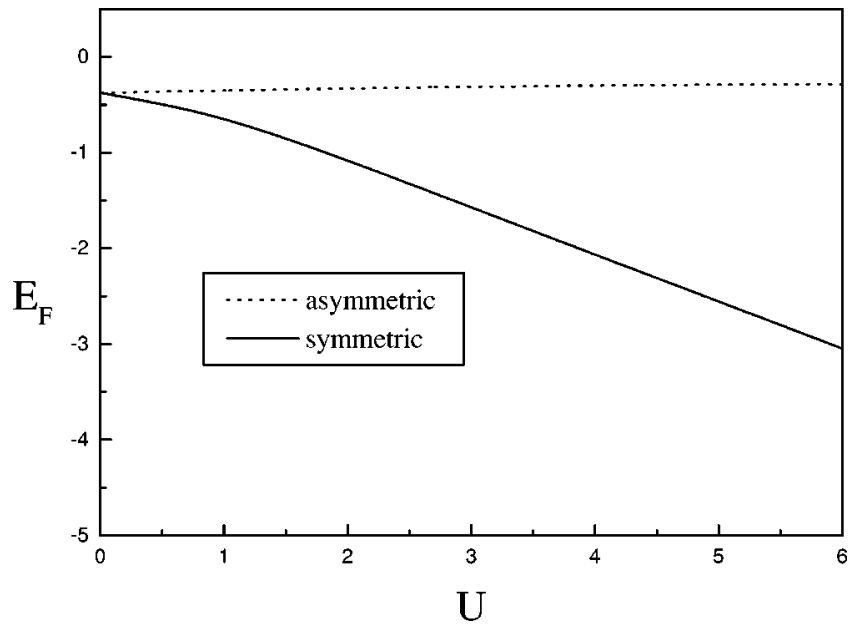

FIG. 10. Fermi energy as a function of $U$ at quarter filling and $V=0.375$, for the symmetric $\left(E_{f}=-U / 2\right)$ and the asymmetric case $\left(E_{f}=0\right)$, respectively.

termediate band filling, there is a second-order transition to a ferromagnetic state, in contrast to the the sharp transition found by Möller and Wölfle. ${ }^{13}$ We find a paramagnetic state at all band fillings for $U<U_{c}$. At quarter filling, there is a transition from a metallic paramagnetic ground state to an insulating antiferromagnetic ordered ground state. Increasing the hybridization stabilizes the ferromagnetic nearestneighbor $f$ - $f$ correlations over a wider range of $U$ and bandfilling values. Overall, the good agreement of our results with the DMRG calculations ${ }^{12}$ and the slave-boson ${ }^{13}$ studies suggests that even though quantum fluctuations are not taken into account, the LMF approach provides a qualitatively correct picture of magnetic ordering in the PAM.

For the asymmetric case in the mixed-valence regime, the ground state is antiferromagnetic at half filling. However, slightly below half filling the ground state becomes ferromagnetic, suggesting that doping away from half filling destroys rapidly the antiferromagnetic $f$ - $f$ spin correlations. For small values of $U$ in the asymmetric case we find, as expected, a paramagnetic state at all fillings. We find a paramagnetic ground state at and slightly above quarter filling $(n=1)$, in agreement with the slave boson results, ${ }^{9}$ but in contradiction with the DMRG results ${ }^{12}$ for the $U=\infty$ case. The square of the local moment in the asymmetric case is suppressed compared to its value in the symmetric case due to charge fluctuations. We have studied the average occupation of the $f$ level and the average of the square of the local moment as a function of $U$ and band filling. At half filling the LMF results are in good agreement with those of DMRG calculations.

Even though the LMF approach is not an exact one, it has the advantage that the method becomes simple enough to generalize its application to the calculation of finitetemperature properties, it can be generalized to the case of higher-dimensional lattices, where only a few results are available, and it allows for the study of all regions in the parameter space.

The work was supported through the National Science Foundation under Grant No. DMR-0097187 and the Office of Research and Sponsored Projects in California State University Northridge.
${ }^{1}$ P. Fulde, J. Keller, and G. Zwicknagl, Solid State Physics, edited by H. Ehrenreich and D. Turnbull (Academic, New York, 1990), Vol. 41, p. 1.

${ }^{2}$ H. R. Ott, in Progress in Low Temperature Physics, edited by D. F. Brewer (North-Holland, Amsterdam, 1987), Vol. XI, p. 215.

${ }^{3}$ C. Kittel, Quantum Theory of Solids (Wiley, New York, 1963).

${ }^{4}$ J. Kondo, Solid State Phys. 23, 183 (1969).

${ }^{5}$ R. Blankenbecler, J. R. Fulco, W. Gill, and D. J. Scalapino, Phys. Rev. Lett. 58, 411 (1987).

${ }^{6}$ M. M. Steiner, R. C. Albers, D. J. Scalapino, and L. J. Sham, Phys. Rev. B 43, 1637 (1991).

${ }^{7}$ K. Ueda, H. Tsunetsugu, and M. Sigrist, Phys. Rev. Lett. 68, 1030 (1992).

${ }^{8}$ T. M. Rice and K. Ueda, Phys. Rev. Lett. 55, 995 (1985); Phys. Rev. B 34, 6420 (1986).
${ }^{9}$ P. S. Riseborough, Phys. Rev. B 45, 13984 (1992); A. Millis and P. A. Lee, ibid. 35, 3394 (1987).

${ }^{10}$ M. Guerrero and C. C. Yu, Phys. Rev. B 51, 10301 (1995).

${ }^{11}$ S. R. White, Phys. Rev. Lett. 68, 3487 (1992); Phys. Rev. B 48, 10345 (1993).

${ }^{12}$ M. Guerrero and R. M. Noack, Phys. Rev. B 53, 3707 (1996).

${ }^{13}$ B. Möller and P. Wölfle, Phys. Rev. B 48, 10320 (1993).

${ }^{14}$ G. Kotliar and A. E. Ruckenstein, Phys. Rev. Lett. 57, 1362 (1986).

${ }^{15}$ T. Yanagisawa, Phys. Rev. Lett. 70, 2024 (1993).

${ }^{16}$ C. Papatriantafillou, N. Kioussis, and S. H. Park, Phys. Rev. B 60, 13355 (1999).

${ }^{17}$ D. M. Newns and N. Read, Adv. Phys. 36, 799 (1987); N. E. Bickers, Rev. Mod. Phys. 59, 845 (1987).

${ }^{18}$ P. W. Anderson, Phys. Rev. 109, 1492 (1958). 